Open Access

\title{
New scoring methodology improves the sensitivity of the Alzheimer's Disease Assessment Scale-Cognitive subscale (ADAS-Cog) in clinical trials
}

Nishant Verma ${ }^{1,2^{*}}$, S. Natasha Beretvas ${ }^{3}$, Belen Pascual ${ }^{4}$, Joseph C. Masdeu ${ }^{4}$, Mia K. Markey ${ }^{1,5}$ and The Alzheimer's Disease Neuroimaging Initiative

\begin{abstract}
Introduction: As currently used, the Alzheimer's Disease Assessment Scale-Cognitive subscale (ADAS-Cog) has low sensitivity for measuring Alzheimer's disease progression in clinical trials. A major reason behind the low sensitivity is its sub-optimal scoring methodology, which can be improved to obtain better sensitivity.

Methods: Using item response theory, we developed a new scoring methodology (ADAS-CogIRT) for the ADAS-Cog, which addresses several major limitations of the current scoring methodology. The sensitivity of the ADAS-CogIRT methodology was evaluated using clinical trial simulations as well as a negative clinical trial, which had shown an evidence of a treatment effect.

Results: The ADAS-Cog was found to measure impairment in three cognitive domains of memory, language, and praxis. The ADAS-CogIRT methodology required significantly fewer patients and shorter trial durations as compared to the current scoring methodology when both were evaluated in simulated clinical trials. When validated on data from a real clinical trial, the ADAS-CogIRT methodology had higher sensitivity than the current scoring methodology in detecting the treatment effect.

Conclusions: The proposed scoring methodology significantly improves the sensitivity of the ADAS-Cog in measuring progression of cognitive impairment in clinical trials focused in the mild-to-moderate Alzheimer's disease stage. This provides a boost to the efficiency of clinical trials requiring fewer patients and shorter durations for investigating disease-modifying treatments.
\end{abstract}

\section{Introduction}

The Alzheimer's Disease Assessment Scale's cognitive subscale (ADAS-Cog) is the standard primary cognitive outcome measure for evaluating treatments in clinical

\footnotetext{
*Correspondence: nishant3115@gmail.com

Data used in preparation of this article were obtained from the Alzheimer's Disease Neuroimaging Initiative (ADNI) database (adni.loni.usc.edu). As such, the investigators within the ADNI contributed to the design and implementation of ADNI and/or provided data but did not participate in analysis or writing of this report. A complete listing of ADNI investigators can be found at: http://adni.loni.usc.edu/wp-content/uploads/how_to_apply/ ADNI_Acknowledgement_List.pdf

'Department of Biomedical Engineering, The University of Texas at Austin, 107 W. Dean Keeton Street Stop C0800, Austin, TX 78712, USA

${ }^{2}$ NeuroTexas Institute Research Foundation, St. David's HealthCare, 1015 E. 32nd Street Suite 404, Austin, TX 78705, USA

Full list of author information is available at the end of the article
}

trials of mild-to-moderate Alzheimer's disease. In patients, the ADAS-Cog measures impairment across several cognitive domains that are considered to be affected early and characteristically in Alzheimer's disease [1]. However, several concerns have been raised recently regarding its sensitivity in measuring progression of cognitive impairment in clinical trials [2-5]. The low sensitivity of the ADAS-Cog has been suggested as a possible reason behind the failure of all clinical trials to date of Alzheimer's disease treatments $[2,3,6,7]$.

The low sensitivity of the ADAS-Cog is primarily due to most of its items suffering from either floor or ceiling effects in different stages of Alzheimer's disease [2, 4, 5, 8]. As a result, the ADAS-Cog is limited in measuring progression of cognitive impairment over the course of 
disease progression. Noting this limitation, research efforts are underway towards modifying the ADAS-Cog and developing new cognitive assessments with better sensitivity $[9,10]$. While the importance of developing better assessments cannot be overstated, their in-depth evaluation and eventual utilization in clinical trials is expected to take a significant amount of time. This opens up a parallel research avenue towards improving the application of the ADAS-Cog in clinical trials, which could help make trials more efficient until a better tool is available.

Another major reason behind the low sensitivity of the ADAS-Cog is its suboptimal scoring methodology, which suffers from low accuracy in measuring cognitive impairment. Currently, cognitive impairment is estimated by simply summing scores across the ADAS-Cog items. This methodology suffers from several limitations. Firstly, the current scoring methodology makes an implicit assumption that a single patient trait is measured by the ADASCog. However, psychometric analysis of the ADAS-Cog has suggested that its items measure impairment in multiple cognitive domains [11-13]. The current scoring methodology is equivalent to a weighted summation of impairment in the cognitive domains measured by the ADAS-Cog. In studies of treatments that improve only a subset of cognitive domains, such as improvement in memory but not in language or praxis, the current methodology obscures the detection of treatment effects [14].

Secondly, the current scoring methodology also implicitly assumes that the levels of cognitive impairment required for answering the ADAS-Cog items incorrectly are uniformly ordered. However, the difficulty levels of the ADAS-Cog items are not uniform [2-4] and most of the total ADAS-Cog scores can actually be achieved by different patterns of scores across the ADAS-Cog items [15]. Moreover, since the ADAS-Cog items vary in their sensitivity to measure the underlying cognitive domains $[2-4,11-13,15]$, an item-level analysis is expected to yield better accuracy in measuring cognitive impairment. An item-level analysis is also significant for addressing psychometric problems of the ADAS-cog (such as item bias due to patient factors), which were not investigated at the time of its design [16]. The current scoring methodology does not allow adjustments for such item-level biases, which leads to unaccounted inter-patient variability and further complicates the detection of treatment effects in clinical trials. A related concern pertains to clinical trials that allow inclusion of patients undergoing symptomatic therapy using acetylcholinesterase inhibitor (AChEI) drugs. AChEI drugs provide short term improvements in cognitive performance, specifically in memoryrelated tasks [17]. If AChEI drugs improve performance on only a subset of the ADAS-Cog items, an item-level analysis may become necessary for isolating the effects of investigative treatments in clinical trials.
Thirdly, the current scoring methodology violates core assumptions of the statistical methods typically employed in clinical trials. The primary efficacy analysis of treatments typically involves linear modeling of serial determinations of the total ADAS-Cog scores of patients using an analysis-of-covariance (ANCOVA) methodology [18-22]. It is reasonable to assume that a patient's true underlying cognitive impairment progresses linearly over short follow-up durations that are typically considered in clinical trials. However, when cognitive impairment is estimated using the total ADAS-Cog scores, linear modeling using the ANCOVA methodology results in correlated errors due to the categorical nature of the ADAS-Cog items $[23,24]$. The ANCOVA methodology assumes errors to be independent and normally distributed, which is violated when the total ADAS-Cog scores are used and results in biased efficacy analysis in trials.

Fourthly, the current scoring methodology lacks a proper definition for the measurement scale of cognitive impairment. This makes comparison and interpretation of cognitive impairment across patients challenging when different variants of the ADAS-Cog are used. In theory, the administration of additional items should only improve measurement precision. However, the current scoring methodology also changes the scale of measurement, with a wider range of scores possible when additional items are administered. The current scoring methodology is also sensitive to missing item responses, scoring errors and variability in the administration of the ADAS-Cog, which are common in clinical trials $[25,26]$.

In this study, we investigated the hypothesis that addressing these limitations associated with the current scoring methodology would improve the sensitivity of the ADAS-Cog in clinical trials. This resulted in a new scoring methodology for the ADAS-Cog based on a comprehensive psychometric analysis using item response theory (ADAS-CogIRT). Some prior studies have investigated the potential of item response theory for scoring the ADASCog and reported very promising preliminary results $[15$, 27]. The ADAS-CogIRT methodology is based on extending this prior work, addressing its limitations, and developing a clinically meaningful scale to measure cognitive impairment. We evaluated the sensitivity of the ADASCog using the ADAS-CogIRT methodology and compared it with the current scoring methodology for detecting treatment effects in clinical trials using simulation experiments and data from a real negative clinical trial [18].

\section{Methods}

Data

The data for this study were assembled from three major public cohorts to ensure that the developed scoring methodology is robust against heterogeneity in patients and study designs. The three cohorts are the Alzheimer's 
Disease Neuroimaging Initiative (ADNI), the Coalition against Major Diseases (CAMD), and the Alzheimer's Disease Cooperative Study (ADCS). These cohorts are briefly described in the Additional file 1: Supplementary Materials. We obtained data from 1,275 participants in ADNI, which included 342 patients clinically diagnosed with probable Alzheimer's disease at baseline, 866 patients diagnosed with amnestic mild cognitive impairment at baseline, and 67 normal controls who converted to amnestic mild cognitive impairment during follow-up. The clinical dementia rating (CDR) scale was used to select mild cognitively impaired patients in ADNI who are likely to be amnestic type. A global CDR score of 0.5 , with at least a 0.5 in the memory domain, was required for inclusion of mild cognitively impaired patients in this study. Out of the 866 mild cognitively impaired patients that satisfied this criterion, 262 converted to a clinical diagnosis of probable Alzheimer's disease during the course of the study [28]. We additionally collected data from 1,828 Alzheimer's patients in the placebo arms of six clinical trials in CAMD and 2,496 Alzheimer's patients in the placebo and treatment arms of six clinical trials in ADCS cohorts. The global CDR scores of Alzheimer's patients across the three cohorts were approximately uniformly distributed between 0.5 (very mild stage), 1 (mild stage), and 2 (moderate stage) points, resulting in good patient heterogeneity with respect to disease severity.

The data consist of longitudinal ADAS-Cog responses over the duration of trial, basic demographics, apolipoprotein-E (APOE) genotype, and details on concomitant treatments of patients. The most common version of the ADAS-Cog, which contains a 'Delayed word recall' item in addition to the original 11 items, was used in this study $[1,29]$. Table 1 summarizes the data from the ADNI and the 12 clinical trials of the CAMD and ADCS cohorts. The data were divided into two subsets. The first subset was used for a comprehensive psychometric analysis of the ADAS-Cog and contained data from the ADNI and the placebo arms of all clinical trials except the trial of huperzine A [18]. For psychometric analysis, data from a single visit of every patient was randomly selected to avoid correlated ADAS-Cog responses. The second subset was used to evaluate the scoring methodology we describe in this paper and contained data from the treatment arms of 11 clinical trials. In addition, the clinical trial of huperzine A, which detected a marginally significant treatment effect [18], was used exclusively to evaluate the sensitivity of the new scoring methodology in a real clinical trial scenario.

The patient data from the ADNI, ADCS, and CAMD cohorts were deidentified before transfer to The University of Texas at Austin. A study specific protocol for the collection and the analysis of deidentified data in this research was approved by the Institutional Review Board of The University of Texas at Austin. Since the data are publicly available and deidentified, ethics approval was not necessary from all the participating institutions in the ADNI, ADCS, and the CAMD cohorts for conducting this research. However, as part of the study protocols for data collection in the three cohorts, all participating institutions obtained ethics approval from their respective institutional review boards in accordance with the Good Clinical Practice guidelines, the Declaration of Helsinki, US 21CFR Part 50-Protection of Human Subjects, and Part 56-Institutional Review Boards, and pursuant to state and federal HIPAA regulations. Written informed

Table 1 Data description: summary of patient characteristics from the ADNI and the clinical trials of the CAMD and ADCS cohorts

\begin{tabular}{|c|c|c|c|c|c|}
\hline Study & Sample size & Gender (\% Females) & APOE (\% ع4 positive) & ADAS-Cog ${ }^{a}$ & Study duration \\
\hline ADNI & 1275 & 41.7 & 58.7 & $14.2 \pm 8.5$ & 8 years \\
\hline CAMD-1105 & 325 & 51.0 & - & $25.2 \pm 12.2$ & 20 months \\
\hline CAMD-1131 & 57 & 59.6 & - & $20.5 \pm 3.6$ & 24 weeks \\
\hline CAMD-1132 & 412 & 43.4 & 38.0 & $19.1 \pm 3.1$ & 51 weeks \\
\hline CAMD-1140 & 137 & 42.3 & - & $19.1 \pm 3.4$ & 24 weeks \\
\hline CAMD-1141 & 492 & 55.3 & - & $9.9 \pm 6.0$ & 23 months \\
\hline CAMD-1142 & 405 & 56.0 & 64.1 & $25.3 \pm 10.4$ & 18 months \\
\hline ADCS-HU [18] & 210 & 64.4 & 65.2 & $27.1 \pm 10.8$ & 24 months \\
\hline ADCS-DHA [19] & 402 & 52.5 & 57.7 & $23.9 \pm 9.0$ & 18 months \\
\hline ADCS-VN [20] & 300 & 63.1 & 71.3 & $30.1 \pm 9.8$ & 24 months \\
\hline ADCS-HC [21] & 409 & 53.9 & 70.0 & $22.6 \pm 8.6$ & 18 months \\
\hline ADCS-LL [22] & 406 & 59.9 & 55.3 & $23.9 \pm 10.5$ & 18 months \\
\hline ADCS-MCI [67] & 769 & 47.0 & 53.0 & $11.03 \pm 4.2$ & 26 months \\
\hline
\end{tabular}

ADNI Alzheimer's Disease Neuroimaging Initiative, CAMD Coalition against Major Diseases, ADCS Alzheimer's Disease Cooperative Study, APOE apolipoprotein-E, HU Huperzine, DHA Docosahexaenoic Acid, VN Valproate Neuroprotection, HC Homocysteine, LL Simvastatin, MCI Mild Cognitive Impairment

${ }^{a}$ Summary total ADAS-Cog scores are represented as mean \pm standard deviation 
consents were obtained from all subjects and authorized study partners in accordance with local institutional review board guidelines before data collection and studyspecific procedures were conducted. A list of participating institutions that obtained ethics approval is provided in the Acknowledgements section.

\section{Psychometric analysis of the ADAS-Cog}

We used multidimensional item response theory (IRT) to evaluate the psychometric properties of the ADAS-Cog for measuring cognitive impairment over the course of disease progression. Traditionally, IRT has been employed for investigating psychometric properties of scales in social and educational research that measured a single latent trait in respondents. However, with advances in estimation theory [30-32] and computational abilities, multidimensional IRT models have started to gain popularity since most psychological constructs are unavoidably multidimensional in nature [33]. Using IRT, probabilities of patients' responses to the ADAS-Cog items were modeled as functions of patients' underlying cognitive impairment. In psychometric theory, these functions are typically known as the item characteristic functions [33, 34]. Based on the nature of the ADAS-Cog items, patients' responses are recorded as either dichotomous (response as either correct or incorrect) or ordinal (responses rated on Likert scales). The three-parameter logistic (3PL) model was used to model the probability of an incorrect response to the dichotomous ADAS-Cog items, which included lower asymptotes to account for really difficult items [33, 34]. For instance, if an ADAS-Cog item is difficult and is answered incorrectly by a quarter of cognitively normal individuals, the lower asymptote of that ADAS-Cog item will be estimated as 0.25 . On the other hand, the different response categories of the ordinal ADAS-Cog items were modeled using the Samejima's graded response model [35]. In the Samejima's graded response models, boundaries between the consecutive response categories are probabilistically modeled using the two-parameter logistic (2PL) models, which can be subtracted to obtain probabilistic models of individual response categories [35]. The key parameters of the ADAS-Cog item characteristic functions are the item slopes and the item intercepts, which represent important characteristics of the ADASCog items. While the slope represents the sensitivity of an item in discriminating between patients with different levels of cognitive impairment, the item intercept represents the difficulty level of an item (or difficulty levels of different response categories of an item) for Alzheimer's patients. The parameters of the ADAS-Cog item characteristic functions were estimated using the MetropolisHastings Robbins-Monro algorithm during both the exploratory [31] and the confirmatory phases of IRT analysis [32].

\section{Cognitive domains assessed by the ADAS-Cog}

The evaluation of the cognitive domains assessed by the ADAS-Cog in Alzheimer's patients is important not only for its associated clinical significance but also for ensuring the validity of IRT analysis. IRT makes a strong assumption of local item independence, i.e., patients' responses to the ADAS-Cog items are determined solely by their underlying extents of cognitive impairment. The use of an inappropriate set of latent traits violates this key assumption, which severely compromises the validity of inferences from IRT analysis [36]. More importantly, local item dependence results in unreliable estimates of latent traits [36], which have been suggested as more accurate measures of cognitive impairment [15, 27]. Therefore, the use of an appropriate number of latent traits is crucial for an accurate IRT-based psychometric analysis of the ADAS-Cog and estimation of latent traits. For this reason, we first performed a parallel analysis on pair-wise polychoric correlations between the ADAS-Cog item responses $[37,38]$ to determine an upper limit on the number of latent traits to be considered for a more in-depth evaluation [39]. Exploratory IRT models were developed for competing latent trait structures with the number of latent traits ranging from one (unidimensional trait structure) to the upper limit determined by the parallel analysis. No restrictions on item-trait loadings were imposed during the exploratory phase of IRT analysis. For the cases of multidimensional latent trait structures, the item-trait loadings were rotated to oblique solutions (oblimin) with the latent traits allowed to be intercorrelated. The oblique solutions have fewer cross-loadings of items across multiple latent traits, which makes clinical interpretation of latent traits easier. The competing latent trait structures were compared using the following criteria:

1. Model fit: The latent trait structure should have good global and item-level fits to the ADAS-Cog responses. Global fit was assessed using the two standard statistics of root mean squared error of approximation (RMSEA) [40] and Tucker Lewis index (TLI) [41]. The criteria of RMSEA $\leq 0.05$ and $\mathrm{TLI} \geq 0.95$ are required for a good global fit [42]. Item-level fit was assessed using the recommended $S$ - $X^{2}$ statistic, which effectively controls type-I error rates $[43,44]$.

2. Local item independence: The local item independence assumption was tested using the recommended $G^{2}$ statistic, which has high sensitivity in detecting local item dependence [45].

3. Clinical relevance: The individual latent traits should be clinically meaningful constructs that are worth measuring separately. The latent trait structure should be in agreement with the motivation behind the design of the ADAS-Cog items in the original study 
[1]. Moreover, the latent trait structure should also be supported by the current knowledge of the pathological processes underlying Alzheimer's disease.

After determining the most appropriate latent trait structure, a confirmatory IRT model was estimated with a restricted item-trait loadings structure. From the exploratory IRT model corresponding to the most appropriate latent trait structure, only the item-trait loadings greater than 0.2 were allowed in the confirmatory IRT model [46]. Furthermore, for the ADAS-Cog items that cross-loaded on multiple latent traits, the weaker itemtrait loadings that were less than 0.3 were included only if they significantly improved the model fit of the ADAS-Cog items. The model fit and the validity of the local independence assumption were evaluated for the confirmatory IRT model. The confirmatory IRT model was used for subsequent psychometric analysis of the ADAS-Cog.

\section{Measurement invariance of the ADAS-Cog items}

The ADAS-Cog items should show measurement invariance across patients, despite their characteristics. We performed differential item functioning (DIF) [47] analyses to investigate measurement bias in the ADAS-Cog items due to the patient-level factors of gender (men/women), education level (less/greater than 13 years), and APOE- $\varepsilon 4$ genotype (presence/absence of an $\varepsilon 4$ allele). The ADNI, CAMD, and ADCS cohorts contain predominantly nonHispanic Caucasian patients, which did not allow DIF analysis due to racial and ethnic factors. In the DIF analyses, the ADAS-Cog item parameters were estimated separately for patient groups and compared using the Lord's Wald test [34] with the Benjamini and Hochberg false discovery rate correction [48]. The Lord's Wald test was used instead of the traditional likelihood ratio test [49] due to the large number of hypothesis being tested in this study, which makes the likelihood ratio test very computationally intensive. Using large sample sizes as considered in this study, the Lord's Wald test has been shown to be sensitive in detecting measurement invariance and asymptotically equivalent to the likelihood ratio test [50].

We additionally investigated the invariance properties of the ADAS-Cog item characteristic functions with respect to the status of concomitant symptomatic therapy using AChEI drugs (presence/absence). In clinical trials involving heterogeneous patient samples with respect to the status of AChEI drugs, the effects of AChEI drugs should be accounted for during statistical analysis to isolate and accurately evaluate the effects of investigative treatments. If AChEI drugs uniformly affect all the ADAS-Cog items assessing a cognitive domain (such as memory), the inclusion of an interaction term with the corresponding progression rate is sufficient to account for the effects of symptomatic therapy. However, an item-level analysis may become necessary if AChEI drugs affect only a subset of the ADAS-Cog items that measure a cognitive domain. In the absence of an item-level analysis, the treatment effects of the AChEI drugs may be inaccurately modeled leading to a biased evaluation of the investigative treatments in clinical trials. While measurement invariance of the ADAS-Cog item characteristic functions is investigated using the same DIF methodology as discussed earlier, violation of measurement invariance should not be interpreted as measurement bias of the ADAS-Cog items but rather as treatment effects of the AChEI drugs on specific subdomains within the cognitive domains.

Longitudinal invariance of the item characteristic functions across different disease stages was also investigated by comparing item parameters estimated using baseline responses of patients versus using their responses at the 24-month visit, when the disease has significantly progressed. We additionally investigated the extent of sample bias and variance in the ADAS-Cog item characteristic functions due to different patient samples considered for estimation. Sample bias was assessed as the goodness-offit of the item characteristic functions to the ADAS-Cog response data from the treatment arms of ADCS studies, which were not used for parameter estimation. Sample variance was evaluated by conducting 1,000 bootstrap replications of estimation of the item characteristic functions with sample replacement.

\section{Measurement of cognitive impairment in patients ADAS-Cog scoring methodology based on IRT modeling (ADAS-CogIRT)}

We propose a new ADAS-Cog scoring methodology based on psychometric modeling using IRT (ADAS-CogIRT) for more accurate measurement of cognitive impairment. Given a patient's responses to the ADAS-Cog items, the ADAS-CogIRT methodology collectively uses the ADAS-Cog item characteristic functions to measure cognitive impairment via maximum-likelihood estimation, i.e., the latent trait values that have the highest likelihood of producing the observed set of item-wise responses. Based on the DIF analysis, appropriate adjustments were included in the item slopes and the intercepts to ensure measurement invariance across patient characteristics. By default, the latent traits in IRT are estimated with means of zero and standard deviations of one. We defined appropriate measurement scales for cognitive impairment by linearly scaling the latent traits obtained from the maximum likelihood estimation, a technique commonly used in educational testing. The values of the scaling parameters were determined based on fulfilling the following two criteria: (1) scores of cognitive impairment 
in mild-to-moderate Alzheimer's patients should be nonnegative; and (2) scores of cognitive impairment can be rounded off to the nearest integers without loss of precision.

\section{Accuracy of the ADAS-CogIRT methodology for measuring cognitive impairment}

Since the ground truth cognitive impairment is unknown, the accuracy of the ADAS-CogIRT methodology for measuring cognitive impairment cannot be directly evaluated. Therefore, we indirectly evaluated the ADAS-CogIRT methodology by assessing its accuracy to predict future ADAS-Cog responses of patients based on their responses in a few initial visits. Specifically, we used the ADAS-Cog responses at the baseline, 6-month, and 12-month visits of patients belonging to the treatment arms of the five ADCS studies to obtain longitudinal estimates of their cognitive impairment. These estimates were used to predict cognitive impairment and the corresponding total ADAS-Cog scores at the 24-month visit. The accuracy of the ADASCogIRT methodology was calculated using the root mean squared error (RMSE $\mathrm{ADAS}_{\mathrm{A}}$ ) between the observed and the predicted total ADAS-Cog scores at the 24-month visit. The RMSE $\mathrm{ADAS}_{\text {of }}$ the ADAS-CogIRT methodology was compared to the RMSE $E_{\mathrm{ADAS}}$ achieved by using the total ADAS-Cog scores as estimates of cognitive impairment in the initial visits.

\section{Precision of the ADAS-CogIRT methodology for measuring cognitive impairment}

The precision of the ADAS-CogIRT methodology is dependent on the amount of information contributed by the ADAS-Cog items for measuring different levels of cognitive impairment. We calculated the item information functions of the ADAS-Cog items to estimate the precision of the ADAS-CogIRT scoring methodology [46]. A high value for the item information at a given level of cognitive impairment implies that the item measures that level of cognitive impairment with high precision. Conversely, low item information at a given cognitive impairment level represents that the item measures that level of cognitive impairment with low precision. The composite information across all the ADAS-Cog items was used to estimate the expected standard error of measurement of different levels of cognitive impairment using the ADAS-CogIRT methodology.

Improving the sensitivity of the ADAS-Cog in clinical trials Application of the ADAS-CogIRT methodology in clinical trials We propose a generalized mixed-effects approach for using the ADAS-CogIRT methodology in clinical trials. Besides estimating baseline cognitive impairment, this approach estimates the rates of progression in cognitive impairment based on patients' longitudinal ADAS-Cog responses. We assumed linear progression of cognitive impairment in patients because the durations of clinical trials are typically too short ( $\sim 2-3$ years) to observe any complex patterns of disease progression. Significant interpatient variability in baseline cognitive impairment and progression rates is typically observed in clinical trials. While some variability is systematic due to patient-level factors (such as APOE- $\varepsilon 4$ genotype) and treatment effects, random variability across patients is also substantial. Therefore, we modeled baseline cognitive impairment and progression rates as mixed-effects in the model to ensure validity of the key assumptions of efficacy analysis. While the fixed effects modeled systematic variability due to patient factors and treatment effects, the random effects accounted for random variability across patients. We evaluated the sensitivity of the ADAS-CogIRT methodology for detecting treatment effects in clinical trials using simulation experiments and a real clinical trial, which had been reported as negative but which showed some evidence of a treatment effect [18].

\section{Sensitivity analysis using clinical trial simulations}

Clinical trials were simulated to mimic the complexity of real-world clinical trials by including unbalanced patient samples, systematic and random inter-patient variability in cognitive impairment and progression rates, and dropout of patients from clinical trials. The parameters for simulating these characteristics were obtained by analyzing the longitudinal ADAS-Cog data from the placebo arms of ADCS and CAMD trials using a generalized mixed-effects model approach. A Cox proportional hazards model was used for modeling hazard of patient dropout with baseline cognitive impairment, progression rates, and patient-level factors as covariates.

The statistical power of the newly proposed (ADASCogIRT) and the standard ADAS-Cog scoring methodologies for detecting treatment effects was evaluated through two simulation experiments. In the first experiment, their power was evaluated for different sample sizes of $200,400,600,800$, and 1,000 patients considered in clinical trials of fixed 24 months duration. For the second experiment, the sample size was fixed as 400 patients and the statistical power was evaluated for different durations of $12,24,36$, and 48 months. These fixed values were selected based on the average characteristics of past clinical trials. Both experiments were repeated for four hypothetical treatment effects of Cohen's $d=0$ (no effect), 0.2 (mild effect), 0.5 (moderate effect), and 0.8 (large effect) simulated in the treatment arms of clinical trials [51]. The case of no treatment effect evaluated the type-I error rates of the proposed scoring methodology. The follow-up visits in both of the experiments were considered to be biannual during the duration of each trial. The ADAS-Cog responses of patients were simulated using the estimated 
item characteristic functions. The slope and the intercept parameters of the item characteristic functions were randomly perturbed in every trial simulation using the estimated standard errors associated with the parameters. The standard errors represent the expected variability in item parameters if different patient samples were considered for parameter estimation. Therefore, simulating the ADAS-Cog responses using perturbed item parameters resembles real world situations, where patient samples to be analyzed would have different characteristics than the patient sample used for estimating the parameters of the ADAS-CogIRT scoring methodology. The perturbation in the item parameters also reduces the extent of bias involved in our simulation experiments from using the same item characteristic functions for both simulating and analyzing the ADAS-Cog response data.

The simulated ADAS-Cog responses were analyzed using the proposed ADAS-CogIRT and the currently employed analysis-of-covariance (ANCOVA) methodologies. The treatments effects in both of the scoring methodologies were assessed using the $z$-statistic with a Bonferroni correction for multiple comparisons. While a significance level of $\alpha=0.05$ was considered for the ANCOVA methodology, the significance level for investigating treatments effects using the ADAS-CogIRT scoring methodology was pre-specified as $\alpha=0.05 / m$, where $m$ denotes the number of cognitive domains assessed by the ADAS-Cog. In both experiments, 500 clinical trials were simulated for every possible combination of treatment effect, sample size, and trial duration. The statistical power was evaluated as the proportion of clinical trials in which a statistically significant treatment effect on patients' progression rates was detected.

\section{Sensitivity analysis using a real clinical trial}

Besides simulations, we additionally evaluated the sensitivity of the ADAS-CogIRT methodology in a real clinical trial study of huperzine A [18]. In the original negative trial, the higher dose level of $400 \mu \mathrm{g}$ had a marginal effect ( $\mathrm{p}$-value $=0.07$ ) on patients' cognitive functioning after 16 weeks [18]. Given this trend from the original ANCOVA analysis, we were interested in determining whether a more sensitive methodology would change the significance of the treatment effect on progression rates of cognitive impairment. Therefore, we re-analyzed the data from the placebo and the $400 \mu g$ huperzine A treatment arms using the ADASCogIRT methodology. The sample size was $141 \mathrm{pa-}$ tients across the two arms in the 16-week long trial. Besides statistical significance, we also calculated the size of treatment effects estimated by the ANCOVA and the ADAS-CogIRT methodologies for a comparison of sensitivities.
All data analyses in this study were performed using the R 3.2.1 software environment for statistical computing. A more detailed description of our Methods is provided in the Additional file 1: Supplementary Material published online only.

\section{Results \\ Psychometric analysis of the ADAS-Cog Cognitive domains assessed by the ADAS-Cog}

From the parallel analysis we estimated that the upper limit on the required number of latent traits is seven. By comparing the latent trait structures for up to seven latent traits using the criteria defined in the 'Cognitive domains assessed by the ADAS-Cog' section, the threedimensional latent trait structure was found to be the most appropriate one. All models with the number of latent traits greater than or equal to three showed a good global and item-level fit to the ADAS-Cog response data. Local item dependence (LID) between a set of items typically indicates that the item set measures additional latent traits besides the traits already considered in the model. The three-dimensional trait structure had LID only between a few subitems, which belong to the same ADAS-Cog items. Since subitems within items tend to share item-specific contexts, such LID is expected. To eliminate all LID, seven traits were required, where several traits were measured only by single items indicating an overfit to the ADAS-Cog response data. We investigated the effect of the presence of LID on the item parameter estimates of the three-dimensional latent trait structure. The item parameter estimates of IRT models with three and seven latent traits were very similar, which suggests that LID in the three-dimensional model is negligible and does not affect item parameter estimates. While lower asymptotes were considered in the item characteristic functions of all the dichotomous ADAS-Cog items, only the constructional praxis subitem assessing a patient's ability to draw a cube was found to have a statistically significant lower asymptote of $20.6 \%$. Drawing a cube is related to the patients' spatial visualization ability, which is known to deteriorate with aging [52]. Therefore, these results indicate that even $20.6 \%$ of cognitively normal elderly people make mistakes in drawing a cube. In the confirmatory IRT model, only the 'Draw a cube' subitem within the 'Constructional Praxis' item was allowed to have a non-zero lower asymptote.

The three-dimensional trait structure also provides a clinically meaningful interpretation. The pattern of dominant item-trait loadings suggests that the three traits basically represent impairment in the memory, language, and praxis cognitive domains (Fig. 1). In the original study by Rosen et al. [1], the ADAS-Cog items were designed to assess these three cognitive domains with the same associations between the items and the domains as 


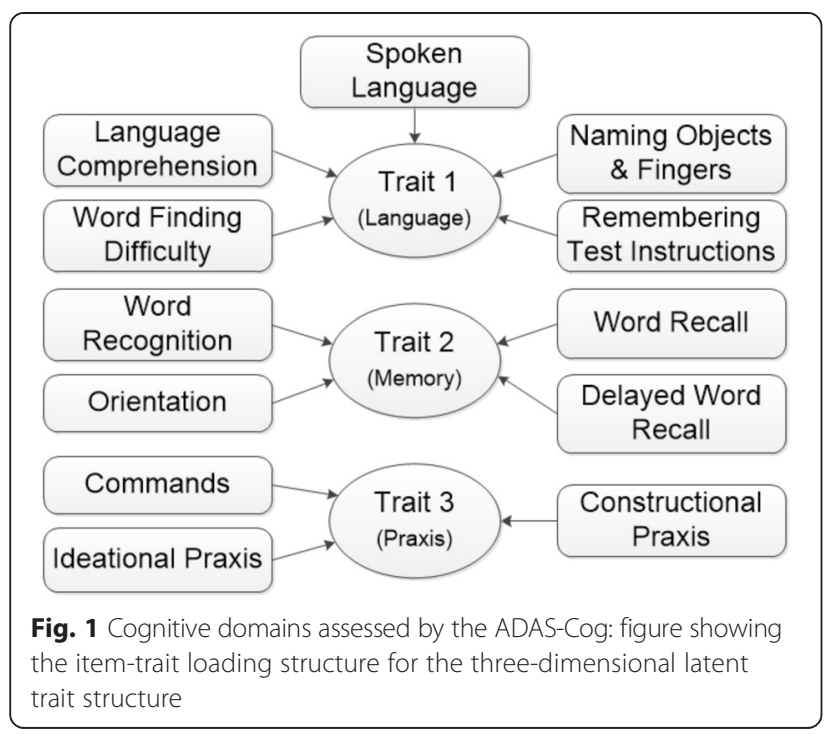

observed in Fig. 1. However, our IRT analysis revealed some additional psychometric properties of the ADAS-Cog items. While the item 'Remembering test instructions' was designed to assess the memory domain [1], psychometric analysis suggests that it cross-loads across both the memory and the language domains, with more dominant loading on the language domain. Similarly, the item 'commands' was designed to assess the language functions in patients. However, psychometric analysis revealed that it cross-loads between the language and the praxis domains, with more dominant loading on the praxis domain.

The three-dimensional latent trait structure is also supported by the underlying neurodegenerative profile of Alzheimer's disease. The classic topography of brain tissue loss in Alzheimer's disease starts early in the medial temporal lobe, which deals with memory functions, followed by involvement of the parietal, frontal, and occipital lobes, which have functions in language processing and praxis [53-56]. A factor analysis of structural brain measurements suggests four distinguishable profiles of neurodegeneration [57], where the brain regions involved in the four profiles are distinctively related to memory, language, and praxis functions. These observations suggest that impairment in the memory, language, and praxis cognitive domains progress differently based on the brain regions involved in different stages of Alzheimer's disease. Therefore, it would be clinically relevant to separately measure cognitive impairment in the memory, language, and praxis domains. The higher dimensional latent trait structures further divided the memory, language, and praxis domains into several subdomains, which were highly inter-correlated $(>0.80)$. For instance, in the seven dimensional latent trait structure, subdomains of semantic memory and working memory were measured by different latent traits. In view of model conciseness and the high correlations between the memory, language, and praxis subdomains, the three dimensional latent trait structure was found to be the most appropriate for measuring cognitive impairment in Alzheimer's patients.

The confirmatory IRT model using the item-trait loading structure in Fig. 1 showed good model fit (RMSEA $=0.039$, $\mathrm{TLI}=0.95$, and $\mathrm{S}-\mathrm{X}^{2}$ insignificant for all the ADAS-Cog items) and low levels of local item dependence as observed in the exploratory IRT model.

\section{Measurement invariance of the ADAS-Cog items}

Table 2 lists the ADAS-Cog items that violate measurement invariance due to patient-level characteristics. Four ADAS-Cog items have measurement bias due to gender because of different item difficulty for men and women. While naming the object "rattle" is easier for women, they are less likely to correctly name "harmonica" and have more difficulty in drawing a cube. A strong measurement bias due to gender was also observed for the item 'Remembering test instructions', where women are more likely to forget test instructions during administration of the ADAS-Cog. No measurement bias was observed due to education level and APOE- $\varepsilon 4$ genotype. AChEI drugs showed treatment effects only on a subset of the ADAS-Cog items that assess the memory domain. Specifically, the item slopes of the 'Word recall,' 'Delayed word recall', and 'Word recognition' items were significantly smaller in patients receiving AChEI drugs. This indicates that patients receiving AChEI drugs have much slower deterioration in their ability to recall and recognize words, which probe short-term working memory. However, other memory-related items (such as 'Orientation') assessing other subdomains of the memory domain were not affected by the use of AChEI drugs.

The ADAS-Cog item parameters estimated using the baseline and the 24-month visit data did not show any statistically significant differences, which suggests that the ADAS-Cog item characteristic functions are longitudinally

Table 2 Differential item functioning: measurement bias of ADAS-Cog items with respect to gender (men/women) and status of concomitant AChEl symptomatic therapy (yes/no)

\begin{tabular}{lll}
\hline DIF factor & ADAS-Cog item & Bias type \\
\hline Gender & Naming objects \& fingers: rattle & $d_{\text {Men }}<d_{\text {Women }^{* * *}}$ \\
Gender & Naming objects \& fingers: harmonica & $d_{\text {Men }}<d_{\text {Women }^{* *}}$ \\
Gender & Constructional Praxis: Cube & $d_{\text {Men }}<d_{\text {Women }^{* *}}$ \\
Gender & Remembering test instructions & $d_{\text {Men }}<d_{\text {Women }}{ }^{* *}$ \\
AChEl & Word recall & $a_{\text {Yes }}<a_{\text {No }}{ }^{* *}$ \\
AChEl & Word recognition & $a_{\text {Yes }}<a_{\text {No }}{ }^{* * *}$ \\
AChEl & Delayed word recall & $a_{\text {Yes }}<a_{\text {No }}{ }^{* * *}$ \\
\hline
\end{tabular}

ADAS-Cog Alzheimer's disease assessment scale-Cognitive subscale, AChEI acetylcholinesterase inhibitors, DIF differential item functioning, $d$ item intercept/ difficulty, $a$ item slope

${ }^{*}$ indicates the level of significance $\left(* *\right.$ for $p$-value $<10^{-4}$ and ${ }^{* * *}$ for $p$-value $<10^{-6}$ ) 
invariant. The item characteristic functions also illustrated little sample bias, with good global (RMSEA $=0.039$ and $\mathrm{TLI}=0.95)$ and item-level fit $\left(S-X^{2}\right.$ was not statistically significant) to response data from the treatment arms of the ADCS clinical trials. The item characteristics functions also showed little variance across different patient samples with a tight agreement observed across 1,000 bootstrap replicates (Additional file 1: Figures S2-S4).

\section{Measurement of cognitive impairment in Alzheimer's patients}

By default, the parameters of the ADAS-Cog item characteristic functions are estimated such that the scores of memory, language, and praxis impairment have means of 0 and standard deviations of 1 in the patient sample. We found that linear scaling by multiplying with factors of 15 and adding 50 points to the scores of memory, language, and praxis impairment were sufficient for satisfying the two criteria of (1) non-negative cognitive impairment scores for mild-to-moderate Alzheimer's patients, and (2) standard errors of magnitude $\sim 1$ point in the mild-to-moderate Alzheimer's stage. Similar approaches have been previously utilized with different scaling factors in the educational and social domains. Instead of scaling the cognitive impairment scores postestimation, we performed an equivalent linearly scaling of the ADAS-Cog item parameters to enforce these measurement scales for estimation of memory, language, and praxis impairment. An additional advantage of the defined measurement scales is the fractional interpretation of the cognitive impairment scores. Severe Alzheimer's patients have cognitive impairment scores close to 100 points and, therefore, a patient's extent of cognitive impairment can be interpreted fractionally relative to severe Alzheimer's disease patients, who have lost the ability to independently function in daily life activities.

\section{Accuracy of the ADAS-CogIRT methodology for measuring cognitive impairment}

The ADAS-CogIRT methodology illustrated good accuracy in predicting total ADAS-Cog scores at the 24-month visit with $\mathrm{RMSE}_{\mathrm{ADAS}}=1.82$ points. In comparison, the current scoring methodology resulted in an error of RMSE $_{\text {ADAS }}=6.05$ points, which is similar in magnitude to the annual change of 5-10 points in the total ADAS-Cog scores of mild-to-moderate Alzheimer's patients $[58,59]$. Figure 2 qualitatively compares the predictive accuracies of the ADAS-CogIRT and the current scoring methodologies.

\section{Precision of the ADAS-CogIRT methodology for measuring cognitive impairment}

While the memory items of the ADAS-Cog are informative over the whole range of memory impairment, language and praxis items hold information only for pronounced levels of language and praxis impairment (Fig. 3a-c). The ADAS-CogIRT methodology shows good precision for almost the whole range of memory impairment. However, due to the inherent limitation of the ADAS-Cog items, the precision of the ADAS-CogIRT methodology in measuring language and praxis impairment is good only when a patient's performance is quite poor (Fig. 3d).

Improving the sensitivity of the ADAS-Cog in clinical trials The treatment effects in the simulated trials and the huperzine A trial were investigated using the ADAS-CogIRT scoring methodology at a significance level of $\alpha=0.05 / 3$
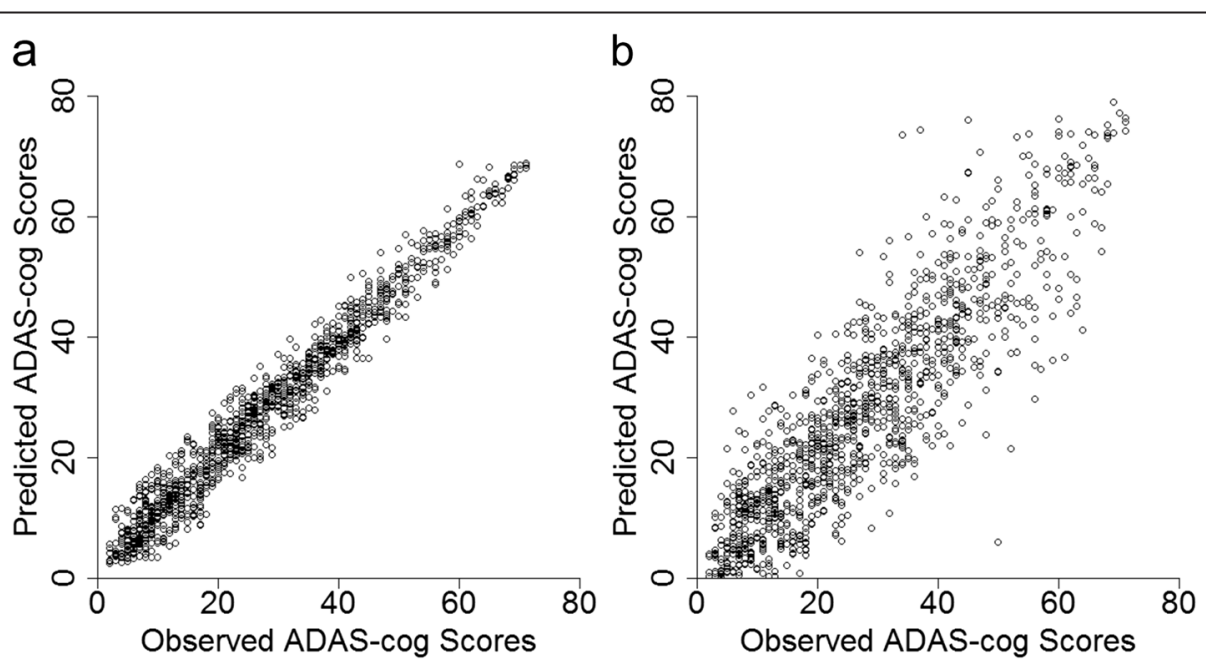

Fig. 2 Accuracy of the ADAS-CogIRT methodology: scatterplots showing agreement between the observed total ADAS-Cog scores and the predicted total ADAS-Cog scores at the 24-month visit using a the proposed ADAS-CogIRT methodology and $\mathbf{b}$ the standard scoring methodology. ADAS-CogIRT Alzheimer's disease assessment scale-Cognitive subscale scoring methodology based on item response theory 

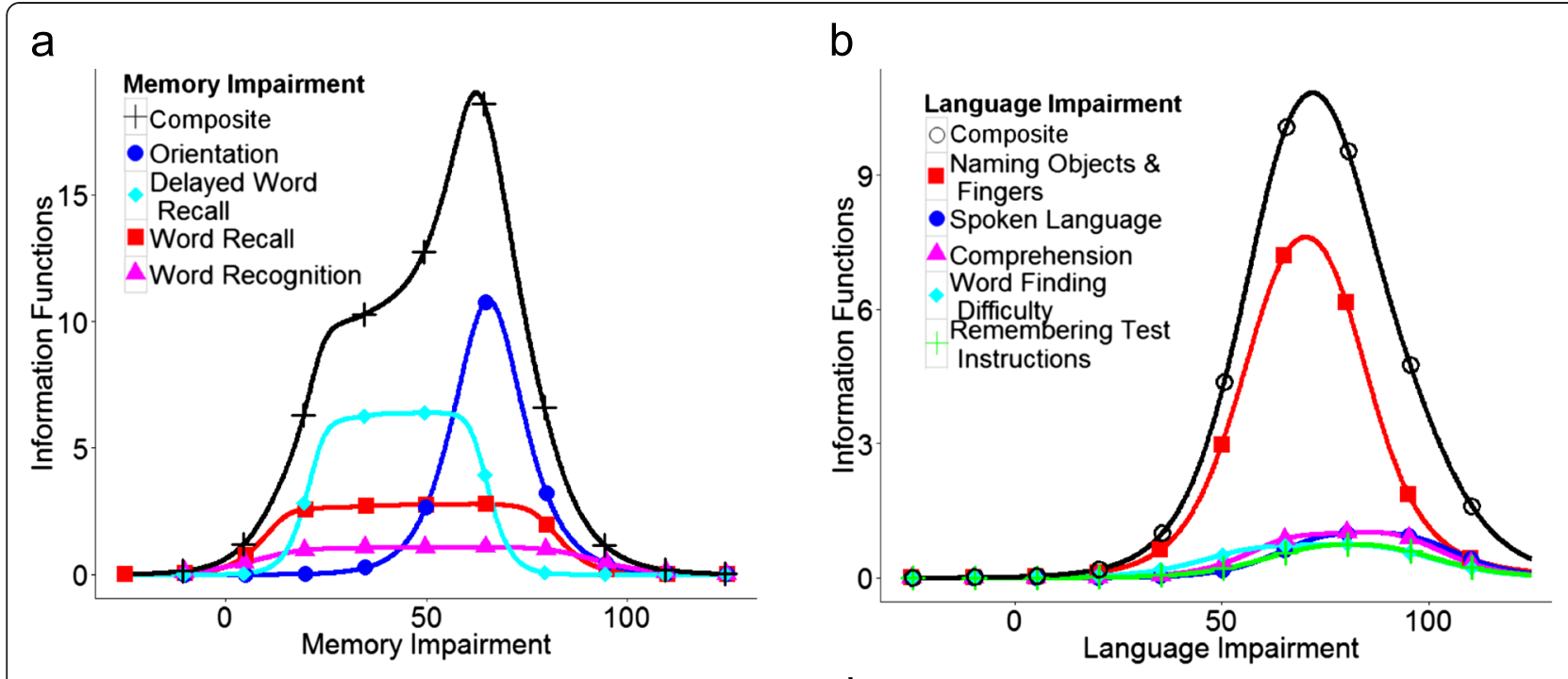

C
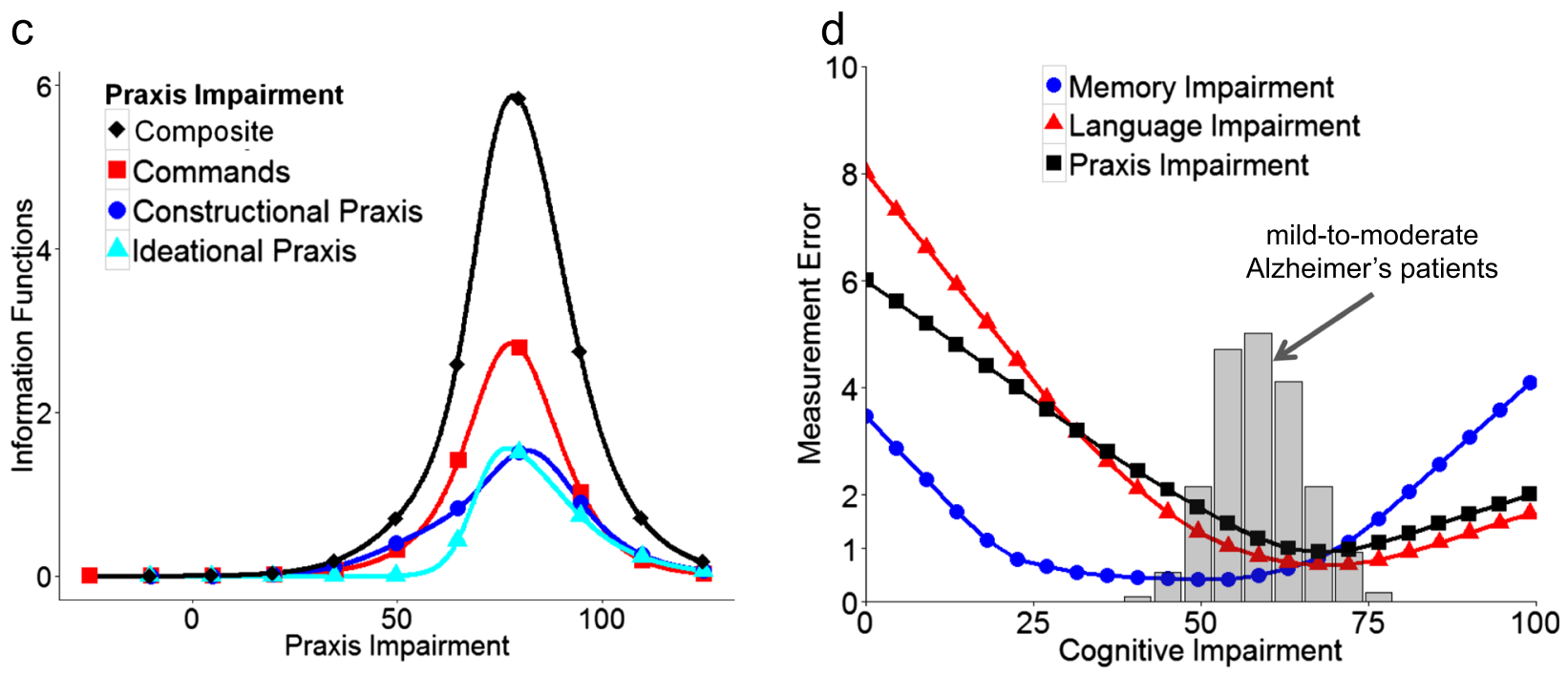

Fig. 3 Precision of the ADAS-CogIRT methodology: figure showing item-wise and cumulative Fisher information associated with the estimation of (a) memory, (b) language, and (c) praxis impairment. The plot in (d) shows the expected magnitudes of estimation errors associated with different levels of memory, language, and praxis impairment. The superimposed histogram in plot (d) shows the distribution of baseline cognitive impairment in mild-to-moderate Alzheimer's patients, which have been appropriately scaled for better interpretation. ADAS-CogIRT Alzheimer's disease assessment scale-Cognitive subscale scoring methodology based on item response theory

since treatments are evaluated in the three domains of memory, language, and praxis simultaneously.

\section{Sensitivity analysis using clinical trial simulations}

In detecting simulated treatment effects, the ADAS-CogIRT methodology provides significant improvements in statistical power over the currently used ANCOVA methodology (Figs. 4b-d and 5b-d). For a mild treatment effect (Figs. $4 \mathrm{~b}$ and $5 \mathrm{~b}$ ), both methodologies have low power and are unable to attain the $80 \%$ power cut-off even with large sample sizes and long trial durations. This is due to large inter-patient variability in progression rates within each trial arm, which obscures the presence of a mild treatment effect. However, in comparison to the
ANCOVA methodology, the ADAS-CogIRT methodology shows better improvements in statistical power as sample size and trial duration are increased (Figs. 4b and $5 \mathrm{~b}$ ). In the case of a moderate treatment effect, the ADAS-CogIRT methodology shows significantly better statistical power than the ANCOVA methodology. The ADAS-CogIRT methodology attains the $80 \%$ power threshold in trials with much smaller sample size $(\sim 300$ patients) and shorter trial duration ( 18 months) than the ANCOVA methodology, which requires $\sim 1,000$ patients in a 24-month trial to achieve $80 \%$ power (Fig. 4c). With a sample size of 400 patients, the ANCOVA methodology never achieves $80 \%$ power even if the trial duration is increased to over four years (Fig. 5c). However, 


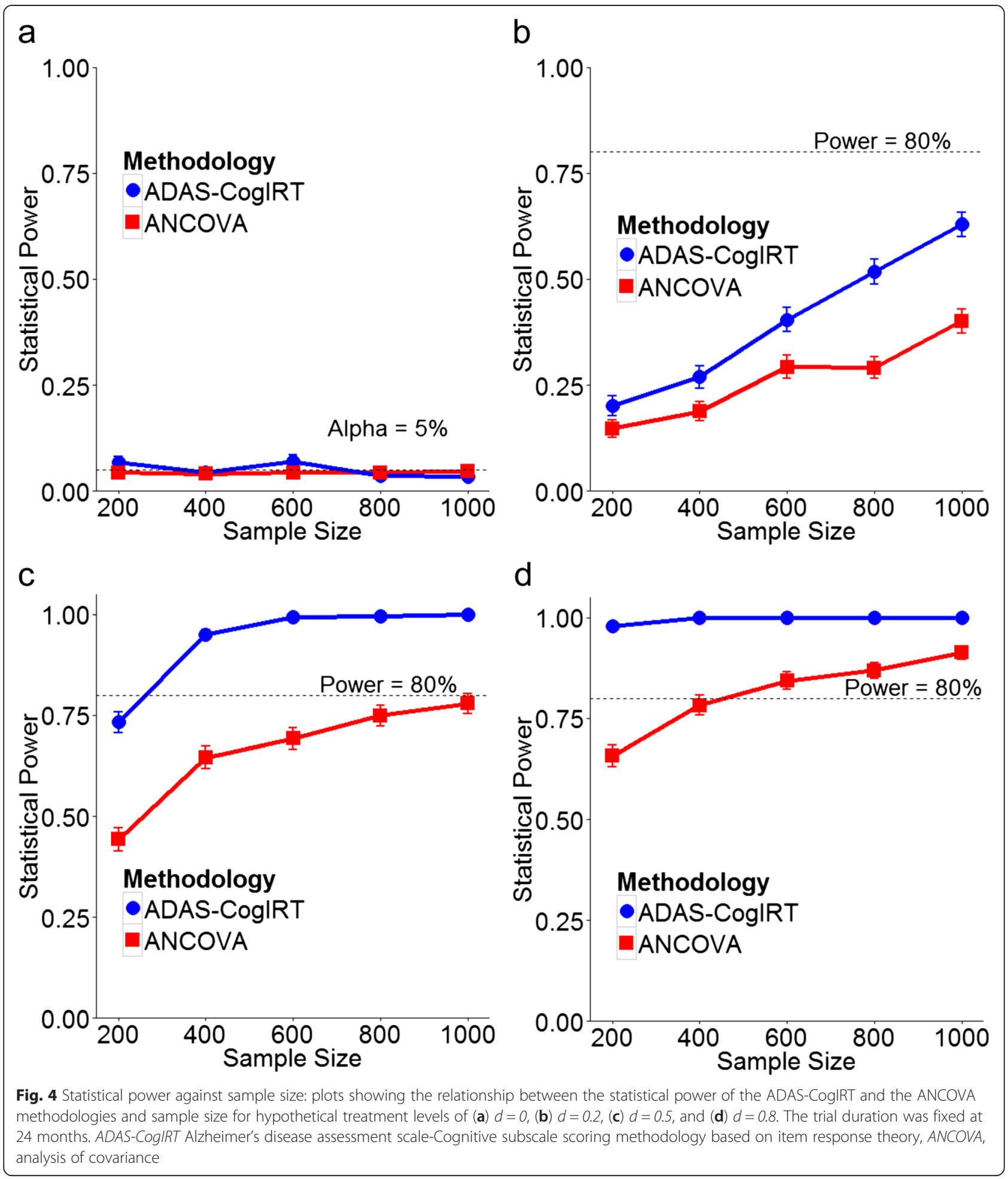

the performance of the ANCOVA methodology improves for a large treatment effect (Figs. 4d and 5d). While the ADAS-CogIRT methodology achieves $~ 100 \%$ power for all sample sizes and trial durations, the ANCOVA methodology also shows good sensitivity reaching $80 \%$ power with $\sim 450$ patients in a 24 -month trial. The improvement in statistical power of both methodologies with an increase in trial duration was less than that observed with an increase in sample size. Both methodologies have acceptable type-1 error rates of $\sim 5 \%$ for different sample sizes and trial durations (Figs. 4a and 5a). 


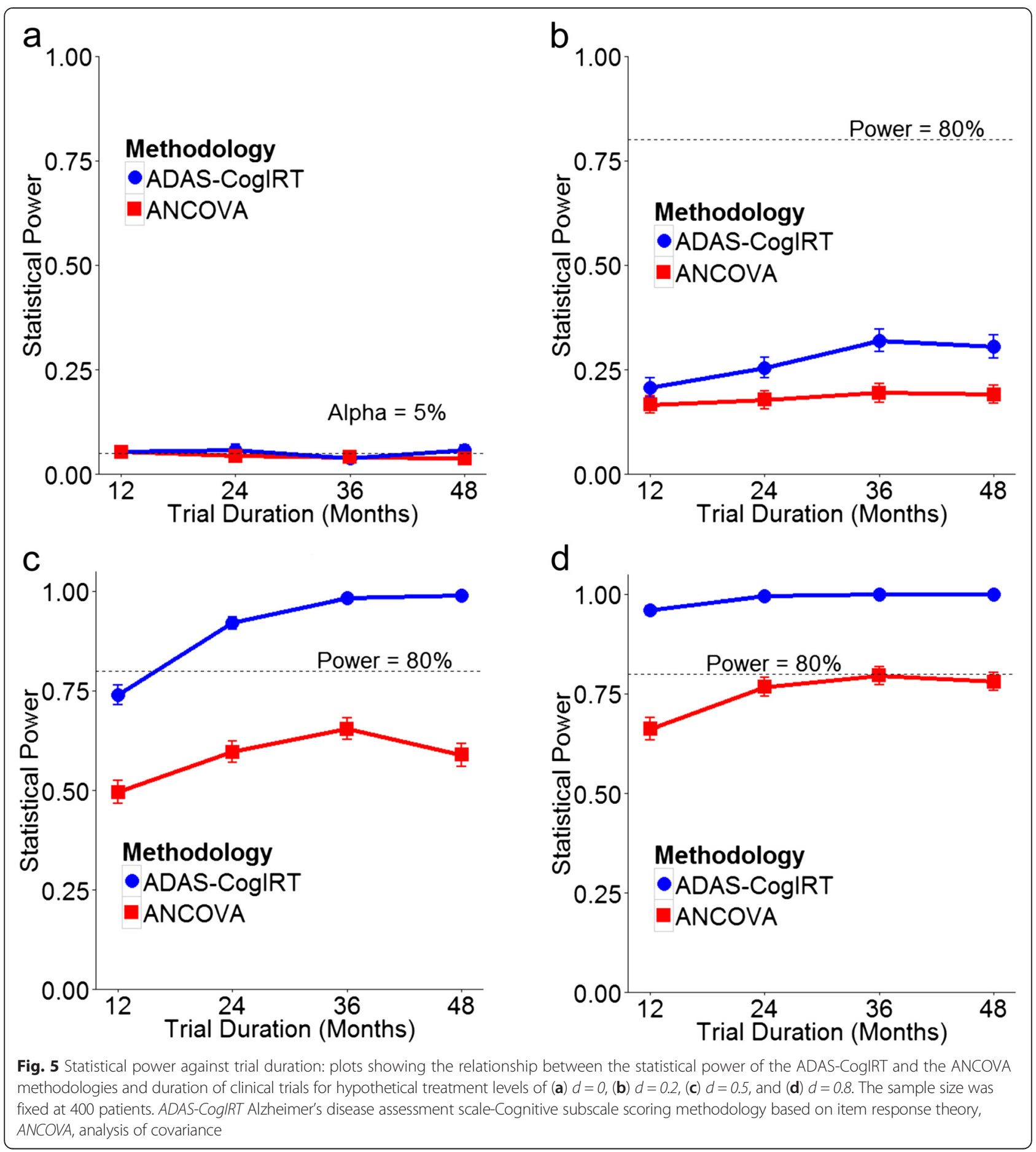

\section{Sensitivity analysis using a real clinical trial}

The analysis of the huperzine A trial data using the ADASCogIRT methodology revealed that $400 \mu g$ huperzine A reduces the annual progression rate of praxis impairment by 14.75 points/year $(z=-2.71, p$-value $=0.0066)$. The effects of huperzine A on progression rates of memory $(z=-1.04, p$-value $=0.30)$ and language impairment $(z$ $=-1.63, p$-value $=0.10$ ) were not statistically significant.
The size of the treatment effect detected by the ADASCogIRT methodology $(d=1.97)$ was significantly higher than that detected by the ANCOVA methodology $(d=$ $0.35)$. Since praxis items contribute the least to the total ADAS-Cog scores (15/70 points), the ANCOVA methodology detects a much smaller treatment effect in comparison to the ADAS-CogIRT methodology. When only the praxis ADAS-Cog items are used in the current scoring 
methodology, the ANCOVA analysis detects a significantly larger treatment effect size $(d=0.68)$, which is statistically significant $(z=-2.49, p$-value $=0.012)$.

\section{Discussion}

The proposed ADAS-CogIRT scoring methodology addresses several limitations associated with the current scoring methodology. An in-depth psychometric analysis showed that the ADAS-Cog measures impairment in the three distinct cognitive domains of memory, language, and praxis in patients. This is in agreement with the design of items in the original ADAS-Cog study [1] and findings of several other factor analysis studies $[11-13,60]$. While memory loss has been long considered characteristic of Alzheimer's disease, its classic neuropathology can also be associated with important language and praxis impairment in patients with predominant posterior perisylvian damage [61]. Similar to AChEI drugs, which specifically target memory mechanisms, and to the effect we detected in the huperzine A trial, investigative treatments in the future may also have non-uniform effects across the three cognitive domains. The current scoring methodology cannot detect non-uniform effects across the cognitive domains. In contrast, the ADAS-CogIRT methodology allows for separate evaluation of treatment effects on the memory, language, and praxis domains.

The ADAS-CogIRT methodology estimates cognitive impairment based on patients' response patterns across the ADAS-Cog items. Such an item-level analysis also allows adjustment for measurement bias of the ADAS-Cog items due to gender. Gender differences in item difficulty are likely due to socio-cultural factors that expose one gender to certain objects and tasks more often than the other gender experiences them. AChEI drugs have a treatment effect on patients' performance on word recall and recognition items. Since these items contribute heavily to the total ADAS-Cog scores (32/80 points), this may be the reason behind the slower cognitive deterioration observed in patients undergoing AChEI drugs, as assessed by the current methodology $[62,63]$. Since only a subset of the ADAS-Cog items assessing the memory domain are affected by the AChEI drugs, adjustments in the item characteristic functions of the three affected ADAS-Cog items are important before application in clinical trials. If such adjustments are not included, the invariance properties of the ADAS-Cog item characteristic functions are violated resulting in an underestimation of cognitive impairment in patients who are taking AChEI drugs. Moreover, the effects of the investigative treatments in clinical trials may be estimated with a positive bias since the treatment effects of the AChEI drugs are underestimated on a domain-level analysis. While including the treatment effects of the AChEI drugs within the ADAS-Cog item characteristic functions may be controversial, we recommend these item-level adjustments in clinical trials since the primary goal is to accurately evaluate the investigative treatments. A domain-level modeling of the AChEI treatment effects is expected to produce biased estimates of the effects of the investigative treatments on the memory domain. Therefore, it makes sense to utilize the established treatment effects of the AChEI drugs within the statistical modeling framework to more accurately investigate the effects of investigative treatments. It should be noted that the experiments presented in this study did not require such item-level adjustments because we did not consider the use of AChEI drugs in simulated clinical trials and the huperzine A trial is homogeneous with respect to AChEI therapy status.

Inspired by the application of IRT in educational testing, we defined a clinically meaningful scale to measure cognitive impairment. In mild-to-moderate Alzheimer's patients, the scale allows estimates to be rounded off to the nearest integers without loss of precision. The scale also facilitates a fractional interpretation of cognitive impairment in study patients, relative to severely impaired patients, who have a cognitive impairment score of 100 points. The parameters of the ADAS-CogIRT methodology are scale independent. Therefore, items can be easily added or removed from the ADAS-CogIRT methodology without having to re-estimate parameters or redefine properties (such as range) of the measurement scale. This is relevant because active research towards improving the ADAS-Cog items is already underway [9]. Since the ADAS-CogIRT methodology pools information across items for estimating cognitive impairment, it is less sensitive to scoring errors in individual items as compared to the current scoring methodology, which is linearly affected. For patients with missing responses to certain items, the ADAS-CogIRT methodology does not require data imputation and estimates cognitive impairment using the set of items answered by the patients. However, measurement precision is lower for patients with missing responses, as would be expected from psychometric theory.

By addressing limitations of the current scoring methodology, the ADAS-CogIRT methodology measures cognitive impairment more accurately (Fig. 2) and makes clinical trials more efficient by reducing the sample size and the follow-up duration required to investigate treatments (Figs. 4 and 5). More importantly, it allows for the detection of treatment effects that may be missed by using the current scoring methodology. This was validated using data from the huperzine A clinical trial, where the ADAS-CogIRT methodology detected a significant improvement in the praxis domain that had been overlooked using the current scoring methodology. The current scoring methodology obscures detection of effects of treatments that only improve a subset of cognitive domains. 
This is evident from the observation that when the ANCOVA analysis is repeated using only the praxis ADAS-Cog items in the current scoring methodology, a statistically significant treatment effect is detected. In agreement with these findings, a positive effect of huperzine A on praxis abilities of patients has been found using the activities of daily living scale $[64,65]$. It is noteworthy that while we assumed linear progression of cognitive impairment in clinical trials, future studies involving longer durations may require models of nonlinear profiles of progression of cognitive impairment. The presented generalized mixed-effects approach for utilizing the ADASCogIRT scoring methodology in clinical trials is flexible and can be extended to include such nonlinear profiles of progression.

Prior work on the application of IRT to the ADAS-Cog mostly focused on evaluating its measurement properties $[4,60,66]$. A few studies additionally investigated IRT for measuring cognitive impairment $[15,27]$; however, they assumed that the ADAS-Cog measures a single trait in patients. While a single trait is easy to interpret and model using IRT, it does not adequately fit patient response data (Additional file 1: Figures S1a-b) and severely violates the core IRT assumption of local item independence, which has severe effects on trait estimates [36]. Similar to the total ADAS-Cog scores, the single trait also measures a weighted average of impairment across multiple cognitive domains. Memory items, which have the highest weights, show the poorest fit to the ADAS-Cog response data (Additional file 1: Figure S1b). As a result, measurement of cognitive impairment from a single latent trait IRT model suffers from low precision and reliability. Despite these shortcomings, a single trait IRT model has been demonstrated to significantly improve the sensitivity of the ADAS-Cog in clinical trial simulations [27]. However, those reported results may be overly optimistic because several of the trial characteristics simulated in the analysis [27] are atypical for real clinical trials, such as frequent follow-ups, no patient dropouts, and no heterogeneity due to patient-level factors. Therefore, for a proper comparison, we additionally evaluated the single trait version of the ADAS-CogIRT methodology in more realistic clinical trial simulations and found it to illustrate significantly lower power than the proposed ADAS-CogIRT methodology (Additional file 1: Figures S5 and S6). Since prior studies were primarily focused on evaluating the potential of IRT in this application domain, they did not define a measurement scale $[15,27]$, resulting in counterintuitive negative scores of cognitive impairment in study patients. As also noted by the authors $[15,27]$, they were additionally limited by ignoring measurement bias and heterogeneity in disease severity of patients.

While our study addressed several limitations of the current scoring methodology, some limitations persist.
Firstly, we could not investigate measurement invariance of the proposed scoring methodology across all patientlevel factors (such as race and ethnicity) due to a lack of heterogeneity in the data. This limitation should be noted in future work in order to avoid biased estimates of cognitive impairment using the ADAS-CogIRT methodology with patient groups not considered in this study. Secondly, when compared to the current scoring methodology, the ADAS-CogIRT methodology requires the use of a computer or a handheld device for measuring cognitive impairment in patients. However, this limitation is less relevant for clinical trials than for routine practice because computing is already required for efficacy analysis of investigative treatments. For routine practice, a specialized application (e.g., for a tablet or phone) could be developed to help providers use the ADAS-CogIRT methodology. Thirdly, the precision of the ADAS-CogIRT methodology for measuring language and praxis impairment is affected by the inherent limitations of the ADAS-Cog items (Fig. 3). As a result, the improvement in sensitivity afforded by the ADAS-CogIRT methodology will decrease for clinical trials focusing on milder stages of Alzheimer's disease. In those disease stages, it may be better to use this tool only for investigating treatment effects on memory impairment. However, this approach would not be applicable to mild Alzheimer's disease patients who have predominant involvement of the parietal lobe [61]. The inclusion of more difficult items probing subtle levels of language and praxis impairment would improve its measurement precision in milder stages of Alzheimer's disease. Fourthly, as is the case for most simulation studies, the evaluation results using simulated clinical trials suffer from some bias. The bias is primarily because the estimated item parameters are used for both simulating patients' response data and within the ADAS-CogIRT scoring methodology for detecting treatment effects. While we reduced the bias by perturbing the ADAS-Cog item parameters (using their estimated standard errors) before simulating patients' ADAS-Cog responses, some bias is still expected.

Despite these limitations, the ADAS-CogIRT methodology holds great significance for clinical trials of Alzheimer's treatments. A significant proportion of clinical trials still focus on the mild-to-moderate disease stages due to the inability to detect Alzheimer's disease early with high specificity. The proposed scoring methodology significantly improves the efficiency of clinical trials focused on the mild-to-moderate stages of Alzheimer's disease. Such an improvement in efficiency of clinical trials is highly desirable for rapid testing of future treatments in the critical quest for a disease-modifying treatment. The ADAS-CogIRT methodology also allows separate evaluation of treatment effects in the memory, language, and praxis domains, which can potentially provide additional 
information on the pharmacological properties of treatments and facilitate development of improved therapies. Future clinical trials of Alzheimer's treatments should consider the proposed ADAS-CogIRT scoring methodology as part of their secondary efficacy analysis to further evaluate and establish the significance of the proposed methodology in comparison to the current scoring methodology.

\section{Conclusions}

The sensitivity of the Alzheimer's disease assessment scale-cognitive subscale (ADAS-Cog) in its current form can be significantly improved by addressing limitations associated with its scoring methodology. In this study, we described a new scoring methodology for the ADAS-Cog called the ADAS-CogIRT, which addresses several major limitations of the current scoring methodology and significantly improves the sensitivity of the ADAS-Cog in measuring progression in cognitive impairment in clinical trials. Future clinical trials of Alzheimer's diseasemodifying treatments should consider the application of the described scoring methodology as part of their secondary efficacy analysis to further validate its significance in comparison to the currently employed scoring methodology.

\section{Additional file}

Additional file 1: Supplementary materials: A supplementary document provides details on the methods required for reproducing the results reported in this paper. The supplementary material also contains some additional statistical results, which have not been included in the paper. (PDF $2056 \mathrm{~kb}$ )

\section{Abbreviations}

2PL: 2 parameter logistic; 3PL: 3 parameter logistic; AChEl: Acetylcholinesterase inhibitors; ADAS-Cog: Alzheimer's disease assessment scale-Cognitive subscale; ADAS-CogIRT: ADAS-Cog scoring methodology based on item response theory; ADCS: Alzheimer's Disease Cooperative Study; ADNI: Alzheimer's Disease Neuroimaging Initiative; ANCOVA: Analysis of covariance; APOE: Apolipoprotein-E; CAMD: Coalition Against Major Diseases; CDR: Clinical dementia rating; DIF: Differential item functioning; IRT: Item response theory; LID: Local item dependence; RMSEA: Root mean squared error of approximation; TLI: Tucker Lewis index.

\section{Competing interests}

The authors declare that they have no competing interests.

\section{Authors' contributions}

All authors (NV, NB, BP, JCM and MKM) made significant contributions to the design of the study and drafting the manuscript. NV and MKM had access to the entire dataset. NV, NB and MKM performed the statistical analysis and simulations of clinical trials. NV, BP, JCM and MKM contributed towards clinical interpretation of results, experiment planning and effective presentation of results in the manuscript. All authors read and approved the final manuscript.

\section{Acknowledgements}

The authors would like to sincerely thank the reviewers for their insightful comments on the paper, which significantly improved the presentation and the quality of this research work. The authors also acknowledge the Texas Advanced Computing Center (https://www.tacc.utexas.edu/) at The
University of Texas at Austin for providing high performance computing resources that were used for conducting the trial simulation experiments reported in this paper.

The collection of a subset of the data used in this study was funded by the Alzheimer's Disease Neuroimaging Initiative (ADNI) (National Institutes of Health Grant U01 AG024904) and DOD ADNI (Department of Defense award number W81XWH-12-2-0012). ADNI is funded by the National Institute on Aging, the National Institute of Biomedical Imaging and Bioengineering, and through generous contributions from the following: Alzheimer's Association; Alzheimer's Drug Discovery Foundation; Araclon Biotech; BioClinica, Inc.; Biogen Idec Inc.; Bristol-Myers Squibb Company; Eisai Inc.; Elan Pharmaceuticals, Inc.; Eli Lilly and Company; Eurolmmun; F. Hoffmann-La Roche Ltd and its affiliated company Genentech, Inc.; Fujirebio; GE Healthcare; IXICO Ltd.; Janssen Alzheimer Immunotherapy Research \& Development, LLC; Johnson \& Johnson Pharmaceutical Research \& Development LLC.; Medpace, Inc.; Merck \& Co., Inc.; Meso Scale Diagnostics, LLC; NeuroRx Research; Neurotrack Technologies; Novartis Pharmaceuticals Corporation; Pfizer Inc.; Piramal Imaging; Servier; Synarc Inc.; and Takeda Pharmaceutical Company. The Canadian Institutes of Health Research is providing funds to support ADNI clinical sites in Canada. Private sector contributions are facilitated by the Foundation for the National Institutes of Health (http://fnih.org/fnih/). The grantee organization is the Northern California Institute for Research and Education, and the study is coordinated by the Alzheimer's Disease Cooperative Study at the University of California, San Diego. ADNI data are disseminated by the Laboratory for Neuro Imaging at the University of Southern California. Ethics approval was obtained from the institutional review boards of each institution involved: Oregon Health and Science University; University of Southern California; University of California San Diego; University of Michigan; Mayo Clinic, Rochester; Baylor College of Medicine; Columbia University Medical Center; Washington University, St. Louis; University of Alabama Birmingham; Mount Sinai School of Medicine; Rush University Medical Center; Wien Center; Johns Hopkins University; New York University; Duke University Medical Center; University of Pennsylvania; University of Kentucky; University of Pittsburgh; University of Rochester Medical Center; University of California, Irvine; University of Texas Southwestern Medical School; Emory University; University of Kansas, Medical Center; University of California, Los Angeles; Mayo Clinic, Jacksonville; Indiana University; Yale University School of Medicine; McGill University, Montreal-Jewish General Hospital; Sunnybrook Health Sciences, Ontario; U.B.C. Clinic for AD \& Related Disorders; Cognitive Neurology - St. Joseph's, Ontario; Cleveland Clinic Lou Ruvo Center for Brain Health; Northwestern University; Premiere Research Institution (Palm Beach Neurology); Georgetown University Medical Center; Brigham and Women's Hospital; Stanford University; Banner Sun Health Research Institute; Boston University; Howard University; Case Western Reserve University; University of California, Davis - Sacramento; Neurological Care of CNY; Parkwood Hospital; University of Wisconsin; University of California, Irvine; Banner Alzheimer's Institute; Dent Neurologic Institute; Ohio State University; Albany Medical College; Hartford Hospital, Olin Neuropsychiatry Research Center; Dartmouth-Hitchcock Medical Center; Wake Forest University Health Sciences; Rhode Island Hospital; Butler Hospital; University of California San Francisco; Medical University South Carolina; St. Joseph's Health Care Nathan Kline Institute; University of lowa College of Medicine; Cornell University and University of South Florida: USF Health Byrd Alzheimer's Institute.

\section{Author details}

${ }^{1}$ Department of Biomedical Engineering, The University of Texas at Austin, 107 W. Dean Keeton Street Stop C0800, Austin, TX 78712, USA. ${ }^{2}$ NeuroTexas Institute Research Foundation, St. David's HealthCare, 1015 E. 32nd Street Suite 404, Austin, TX 78705, USA. ${ }^{3}$ Department of Educational Psychology, The University of Texas at Austin, 1 University Station D5800, Austin, TX 78712, USA. ${ }^{4}$ Nantz National Alzheimer Center, Houston Methodist Neurological Institute, 6560 Fannin Street, Houston, TX 77030, USA. ${ }^{5}$ Department of Imaging Physics, The University of Texas MD Anderson Cancer Center, 1400 Pressler Street FCT14.50000, Houston, TX 77030, USA.

Received: 30 March 2015 Accepted: 28 September 2015 Published online: 26 October 2015

\section{References}

1. Rosen WG, Mohs RC, Davis KL. A new rating scale for Alzheimer's disease. Am J Psychiatry. 1984;141:1356-64. 
2. Cano SJ, Posner HB, Moline ML, Hurt SW, Swartz J, Hsu T, et al. The ADAScog in Alzheimer's disease clinical trials: psychometric evaluation of the sum and its parts. J Neurol Neurosurg Psychiatry. 2010;81:1363-8.

3. Raghavan N, Samtani MN, Farnum M, Yang E, Novak G, Grundman M, et al. The ADAS-Cog revisited: novel composite scales based on ADAS-Cog to improve efficiency in $\mathrm{MCl}$ and early AD trials. Alzheimers Dement. 2013;9:S21-31.

4. Hobart J, Cano S, Posner H, Selnes O, Stern Y, Thomas R, et al. Putting the Alzheimer's cognitive test to the test II: Rasch Measurement Theory. Alzheimers Dement. 2013;9:S10-20.

5. Hobart J, Cano S, Posner H, Selnes O, Stern Y, Thomas R, et al. Putting the Alzheimer's cognitive test to the test I: Traditional psychometric methods. Alzheimers Dement. 2013;9:S4-9.

6. Fleisher AS, Donohue M, Chen K, Brewer JB, Aisen PS. Applications of neuroimaging to disease-modification trials in Alzheimer's disease. Behav Neurol. 2009:21:129-36.

7. Salloway S, Mintzer J, Weiner MF, Cummings JL. Disease-modifying therapies in Alzheimer's disease. Alzheimers Dement. 2008:4:65-79.

8. Grove RA, Harrington CM, Mahler A, Beresford I, Maruff P, Lowy MT, et al. A randomized, double-blind, placebo-controlled, 16-week study of the H3 receptor antagonist, GSK239512 as a monotherapy in subjects with mild-tomoderate Alzheimer's disease. Curr Alzheimer Res. 2014;11:47-58.

9. Skinner J, Carvalho JO, Potter GG, Thames A, Zelinski E, Crane PK, et al. The Alzheimer's Disease Assessment Scale-Cognitive-Plus (ADAS-Cog-Plus): an expansion of the ADAS-Cog to improve responsiveness in $\mathrm{MCl}$. Brain Imaging Behav. 2012;6:489-501.

10. Harrison J, Minassian SL, Jenkins L, Black RS, Koller M, Grundman M. A neuropsychological test battery for use in Alzheimer disease clinical trials. Arch Neurol. 2007:64:1323-9.

11. Talwalker S, Overall JE, Srirama MK, Gracon SI. Cardinal features of cognitive dysfunction in Alzheimer's disease: a factor-analytic study of the Alzheimer's Disease Assessment Scale. J Geriatr Psychiatry Neurol. 1996;9:39-46.

12. Olin JT, Schneider LS. Assessing response to tacrine using the factor analytic structure of the Alzheimer's disease assessment scale (Adas) — cognitive subscale. Int J Geriatr Psychiatry. 1995;10:753-6.

13. Kim YS, Nibbelink DW, Overall JE. Factor structure and reliability of the Alzheimer's Disease Assessment Scale in a multicenter trial with linopirdine. J Geriatr Psychiatry Neurol. 1994;7:74-83.

14. Weintraub D, Somogyi M, Meng X. Rivastigmine in Alzheimer's disease and Parkinson's disease dementia: an ADAS-cog factor analysis. Am J Alzheimers Dis Other Demen. 2011:26:443-9.

15. Balsis S, Unger AA, Benge JF, Geraci L, Doody RS. Gaining precision on the Alzheimer's Disease Assessment Scale-cognitive: a comparison of item response theory-based scores and total scores. Alzheimers Dement. 2012;8:288-94

16. Crane PK, van Belle G, Larson EB. Test bias in a cognitive test: differential item functioning in the CASI. Stat Med. 2004;23:241-56

17. Persson CM, Wallin AK, Levander S, Minthon L. Changes in cognitive domains during three years in patients with Alzheimer's disease treated with donepezil. BMC Neurol. 2009;9:7.

18. Rafii MS, Walsh S, Little JT, Behan K, Reynolds B, Ward C, et al. A phase II trial of huperzine A in mild to moderate Alzheimer disease. Neurology. 2011;76:1389-94

19. Quinn JF, Raman R, Thomas RG, Yurko-Mauro K, Nelson EB, Van Dyck C, et al. Docosahexaenoic acid supplementation and cognitive decline in Alzheimer disease: a randomized trial. JAMA. 2010;304:1903-11.

20. Tariot PN, Schneider LS, Cummings J, Thomas RG, Raman R, Jakimovich LJ, et al. Chronic divalproex sodium to attenuate agitation and clinical progression of Alzheimer disease. Arch Gen Psychiatry. 2011;68:853-61.

21. Aisen PS, Schneider LS, Sano M, Diaz-Arrastia R, van Dyck CH, Weiner MF, et al. High-dose B vitamin supplementation and cognitive decline in Alzheimer disease: a randomized controlled trial. JAMA. 2008;300:1774-83.

22. Sano M, Bell KL, Galasko D, Galvin JE, Thomas RG, van Dyck CH, et al. A randomized, double-blind, placebo-controlled trial of simvastatin to treat Alzheimer disease. Neurology. 2011;77:556-63.

23. Samtani MN, Raghavan N, Shi Y, Novak G, Farnum M, Lobanov V, et al. Disease progression model in subjects with mild cognitive impairment from the Alzheimer's disease neuroimaging initiative: CSF biomarkers predict population subtypes. Br J Clin Pharmacol. 2013;75:146-61.

24. Samtani MN, Farnum M, Lobanov V, Yang E, Raghavan N, DiBernardo A, et al. An improved model for disease progression in patients from the Alzheimer's disease neuroimaging initiative. J Clin Pharmacol. 2012;52:629-44.
25. Schafer K, De Santi S, Schneider LS. Errors in ADAS-cog administration and scoring may undermine clinical trials results. Curr Alzheimer Res. 2011;8:373-6.

26. Connor DJ, Sabbagh MN. Administration and scoring variance on the ADAS-Cog. J Alzheimers Dis. 2008;15:461-4.

27. Ueckert S, Plan EL, Ito K, Karlsson M, Corrigan B, Hooker AC. Improved utilization of ADAS-cog assessment data through item response theory based pharmacometric modeling. Pharm Res. 2014;31:2152-65.

28. McKhann G, Drachman D, Folstein M, Katzman R, Price D, Stadlan EM. Clinical diagnosis of Alzheimer's disease: report of the NINCDS-ADRDA Work Group under the auspices of Department of Health and Human Services Task Force on Alzheimer's Disease. Neurology. 1984;34:939.

29. Mohs RC, Knopman D, Petersen RC, Ferris SH, Ernesto C, Grundman M, et al. Development of cognitive instruments for use in clinical trials of antidementia drugs: additions to the Alzheimer's Disease Assessment Scale that broaden its scope. Alzheimer Dis Assoc Disord. 1997;11:13-21.

30. Bock RD, Aitkin M. Marginal maximum likelihood estimation of item parameters: application of an EM algorithm. Psychometrika. 1981;46:443-59.

31. Cai L. High-dimensional exploratory item factor analysis by a MetropolisHastings Robbins-Monro algorithm. Psychometrika. 2010;75:33-57.

32. Cai L. Metropolis-Hastings Robbins-Monro algorithm for confirmatory item factor analysis. J Educ Behav Stat. 2010;35:307-35.

33. Reckase MD. Multidimensional item response theory. New York: Springer; 2009.

34. Lord FM. Applications of item response theory to practical testing problems. London: Routledge; 1980.

35. Samejima F. Graded response model. In: Handbook of modern item response theory. New York: Springer; 1997. p. 85-100.

36. Zenisky AL, Hambleton RK, Sireci SG. Effects of local item dependence on the validity of IRT item, test, and ability statistics. MCAT Monograph. Association of American Medical Colleges, Section for the Medical College Admission TestMonograph number: 5. 2001.

37. Horn $J$ L. A rationale and test for the number of factors in factor analysis. Psychometrika. 1965;30:179-85.

38. Henson RK, Roberts JK. Use of exploratory factor analysis in published research common errors and some comment on improved practice. Educ Psychol Meas. 2006;66:393-416.

39. Wood JM, Tataryn DJ, Gorsuch RL. Effects of under-and overextraction on principal axis factor analysis with varimax rotation. Psychol Methods. 1996;1:354.

40. Cheung GW, Rensvold RB. Evaluating goodness-of-fit indexes for testing measurement invariance. Struct Equ Modeling. 2002;9:233-55.

41. Tucker $L R$, Lewis $C$. A reliability coefficient for maximum likelihood factor analysis. Psychometrika. 1973;38:1-10.

42. Hu L, Bentler PM. Cutoff criteria for fit indexes in covariance structure analysis: conventional criteria versus new alternatives. Struct Equ Modeling. 1999;6:1-55.

43. Orlando M, Thissen D. Further investigation of the performance of S-X2: an item fit index for use with dichotomous item response theory models. Appl Psychol Meas. 2003;27:289-98.

44. Zhang B, Stone CA. Evaluating item fit for multidimensional item response models. Educ Psychol Meas. 2008:68:181-96.

45. Chen $\mathbf{W H}$, Thissen D. Local dependence indexes for item pairs using item response theory. J Educ Behav Stat. 1997;22:265-89.

46. De Ayala RJ. Theory and practice of item response theory. New York Guilford Publications; 2013.

47. Holland PW, Wainer H. Differential item functioning. London: Routledge; 2012

48. Benjamini $Y$, Hochberg $Y$. Controlling the false discovery rate: a practical and powerful approach to multiple testing. J R Stat Soc Series B Methodol. 1995;57:289-300.

49. Holland PW, Wainer H. (eds.) Differential Item Functioning. Hillsdale, NJ: Lawrence Erlbaum Associates, Publishers, 1993:67-113.

50. Langer MM. A reexamination of Lord's Wald test for differential item functioning using item response theory and modern error estimation. Chapel Hill: The University of North Carolina; 2008.

51. Cohen J. Statistical power analysis for the behavioral sciences. New York: Academic Press; 2013.

52. Salthouse TA, Babcock RL, Skovronek E, Mitchell DR, Palmon R. Age and experience effects in spatial visualization. Dev Psychol. 1990;26:128.

53. Frisoni GB, Henneman WJ, Weiner MW, Scheltens P, Vellas B, Reynish E, et al The pilot European Alzheimer's disease neuroimaging initiative of the European Alzheimer's disease consortium. Alzheimers Dement. 2008:4:255-64.

54. Thompson PM, Hayashi KM, de Zubicaray G, Janke AL, Rose SE, Semple J, et al. Dynamics of gray matter loss in Alzheimer's disease. J Neurosci. 2003;23:994-1005. 
55. Scahill Rl, Schott JM, Stevens JM, Rossor MN, Fox NC. Mapping the evolution of regional atrophy in Alzheimer's disease: unbiased analysis of fluid-registered serial MRI. Proc Natl Acad Sci U S A. 2002;99:4703-7.

56. McDonald CR, McEvoy LK, Gharapetian L, Fennema-Notestine C, Hagler DJ, Holland D, et al. Regional rates of neocortical atrophy from normal aging to early Alzheimer disease. Neurology. 2009;73:457-65

57. Desikan RS, Cabral HJ, Settecase F, Hess CP, Dillon WP, Glastonbury CM, et al. Automated MRI measures predict progression to Alzheimer's disease. Neurobiol Aging. 2010;31:1364-74.

58. Beckett LA, Harvey DJ, Gamst A, Donohue M, Kornak J, Zhang H, et al. The Alzheimer's Disease Neuroimaging Initiative: annual change in biomarkers and clinical outcomes. Alzheimers Dement. 2010;6:257-64.

59. Stern RG, Mohs RC, Davidson M, Schmeidler J, Silverman J, Kramer-Ginsberg $\mathrm{E}$, et al. A longitudinal study of Alzheimer's disease: measurement, rate, and predictors of cognitive deterioration. Am J Psychiatry. 1994;151:390-6.

60. Verma N, Markey MK: Item response analysis of Alzheimer's disease assessment scale. In 36th Annual International Conference of the IEEE Engineering in Medicine and Biology Society. IEEE; 2014:2476-2479.

61. Whitwell JL, Dickson DW, Murray ME, Weigand SD, Tosakulwong N, Senjem $M L$, et al. Neuroimaging correlates of pathologically defined subtypes of Alzheimer's disease: a case-control study. Lancet Neurol. 2012;11:868-77.

62. Rösler M, Anand R, Cicin-Sain A, Gauthier S, Agid Y, Dal-Bianco P, et al. Efficacy and safety of rivastigmine in patients with Alzheimer's disease. BMJ. 1999;318:633-40.

63. Rogers SL, Farlow MR, Doody RS, Mohs R, Friedhoff LT. A 24-week, doubleblind, placebo-controlled trial of donepezil in patients with Alzheimer's disease. Neurology. 1998:50:136-45.

64. Wang $B$, Wang H, Wei Z, Song Y, Zhang L, Chen H. Efficacy and safety of natural acetylcholinesterase inhibitor huperzine $A$ in the treatment of Alzheimer's disease: an updated meta-analysis. J Neural Transm. 2009;116:457-65

65. Li J, Wu HM, Zhou RL, Liu GJ, Dong BR. Huperzine A for Alzheimer's disease. Cochrane Database Syst Rev. 2008;2, CD005592.

66. Benge JF, Balsis S, Geraci L, Massman PJ, Doody RS. How well do the ADAScog and its subscales measure cognitive dysfunction in Alzheimer's disease? Dement Geriatr Cogn Disord. 2009;28:63.

67. Petersen RC, Thomas RG, Grundman M, Bennett D, Doody R, Ferris S, et al. Vitamin $E$ and donepezil for the treatment of mild cognitive impairment. $N$ Engl J Med. 2005;352:2379-88.

\section{Submit your next manuscript to BioMed Central and take full advantage of:}

- Convenient online submission

- Thorough peer review

- No space constraints or color figure charges

- Immediate publication on acceptance

- Inclusion in PubMed, CAS, Scopus and Google Scholar

- Research which is freely available for redistribution 